\title{
Interactive comment on "Polarization properties of aerosol particles over western Japan: classification, seasonal variation, and implications for air quality" by X. L. Pan et al.
}

\section{Anonymous Referee \#2}

Received and published: 2 March 2016

This paper describes ground based observation of the aerosol by using a novel methodology of Polarization Optical Particle Counter (POPC) in Kyushu, Japan for more than a year. The paper brings a new insight for the ground based aerosol observations and air quality studies by presenting vigorous POPC data analysis as well as the one coupled with trajectory analysis. So, the present reviewer feels the paper being very interesting and worth to be published. Also, the revision before its publishing in ACPD made the paper concise and better.

The method for observation and data analysis seem almost fine. The data set seems valuable. Although the present manuscript is organized very well, there are many technical points to be amended. Therefore, he recommends a revision of the present 
manuscript before its final publication in the Atmospheric Chemistry and Physics journal. The authors are encouraged to do so. In addition, he recommends that the final manuscript could have native speaker's edition.

Overall, the style adjustment and editing should be carefully made.

*Technical - Important information of the observation duration is not described obviously in the Measurements section. At line 13 in page 3, limited information of "beginning in October 2013" appears.

- Line 31 in page 3: "at Kyushu University (Longitude: ..." -> "at Kyushu University, Fukuoka (Longitude: ..."?

- Line 19 in page 4: "at Fukuoka University (Longitude: ..." -> "at Fukuoka University (Longitude: ..."?

- Line 26 in page 4: "... method, after the addition ..." -> ". . method, respectively, the latter being with the addition ..."?

- Lines 22 in page 5: "spring (MAM)" would be better "spring (MAM: May, April and May) "? The same for summer, fall and winter in the manuscript for the first appearance.

- Lines 7-8 in page 6: The argument sounds better when reference literature is added.

- Lines 32-34 in page 6: "a DR-Dp-volume plot" does not exactly match with labels of Figure 5. The vertical label is "Mode DR", however, the above sentence means quite different. In my understanding the label should be "DR" or "Depolarization ratio (DR)". Is this correct? If not, all "DR" should be expressed as "MDR" in the text. This should be applied to the term appearing at the first line in page 7 and so on.

- Line 34 in page 6: "areas" should be in line with the term in Figures ("regions")? Please clarify "regions" or "the Regions" afterwards.

- Line 2 in page 8: Please add literature. 
- Line 5 in page 8: "the winter" -> "winter"? This is depending on if "the winter" means "2014 winter" or not.

- Line 12 in page 8: Please insert "geographical" in "different origins".

- Line 13 in page 8: Please add "(Figure 7)" after ". . trajectory analysis".

Interactive

- Line 22 in page 8: "fine mode" -> "coarse mode"??

comment

- Line 24 in page 8: "North China" -> "northern China"?

- Line 32 in page 8: "Region I" -> "the Region I"? There are many similar ones hereafter.

- Line 34 in page 10: "LRT" should be spelled out.

- Caption of Figure 1: "Spring (MAM)" -> "spring (MAM)" or "spring (March, April and May)".

- All labels and notations in Figure 2 should start from capital (uppercase letter).

- Legend of Figure 3: "... mixed type dominated cases" -> ". . mixed type dominated cases, respectively". Also, the observation duration should be written obviously in figure caption.

- The above mentioned points should be applied to Figure 4.

- Caption and labels of Figure 5: Please check "MDR" or "DR". "size" -> "size (Dp)"?

- Figure 7: Number of the trajectories analyzed should be described obviously.

- Caption of Figure 7: "Air mass originating" -> "Air mass arrived at Fukuoka, Japan, which originating"?

- Caption of Figure 9: "NH4+" "4" should be lowercase letter.

- Figures 6, 7 and 8 are in low resolution.

Interactive comment on Atmos. Chem. Phys. Discuss., doi:10.5194/acp-2015-904, 2016. 